\title{
Decalepis hamiltonii (Swallow Root) as a Potential Antimicrobial Agent against Endodontic Pathogens: An In Vitro Study
}

\author{
Anand V Susila ${ }^{1}$, Selvam Sangeeta ${ }^{2}$
}

\begin{abstract}
Aim and objective: The aim of the article was to evaluate and compare the antimicrobial effect of alcoholic and hydroalcoholic extracts of Decalepis hamiltonii with those of Curcuma longa, Azadirachta indica, and Zingiber officinale against Enterococcus faecalis.

Materials and methods: Alcoholic and hydroalcoholic extracts of the herbs were prepared by cold maceration and filtration-decantation process. Minimum bactericidal concentration (MBC) and minimum inhibitory concentration (MIC) were determined for each extract; zone of inhibition (ZOI) was assessed separately for each extract and their different combinations on both laboratory strain and clinical isolates of $E$. faecalis.

Results: The extracts of $D$. hamiltonii showed a significant antimicrobial action, against endodontic pathogen $E$. faecalis, both independently and in combination preparations.

Conclusion: Extract of $D$. hamiltonii can be used as intracanal irrigant and medicament in endodontics after confirming its biocompatibility. Keywords: Antimicrobial activity, Decalepis hamiltonii, Endodontic disinfection, Enterococcus faecalis, Herbal extracts, Natural products. Journal of Operative Dentistry and Endodontics (2021): 10.5005/jp-journals-10047-0107
\end{abstract}

\section{INTRODUCTION}

Natural products have played a significant role in eradicating infectious human diseases since time immemorial. There has been a renewed interest and exploration of natural remedies in modern medicine particularly in the last two decades. ${ }^{1-4}$ The main goal of endodontics is to eradicate infectious material from canal space and ensure the healing of periapical tissues without reinfection. Chemical and mechanical methods have been popular in achieving this end. Irrigants form the mainstay of the chemical method of debridement and disinfection in endodontics. ${ }^{5,6}$ The irrigants are required to possess antiseptic, bacteriostatic/ bactericidal properties without periapical tissue irritation in the event of inadvertent extrusion. Conventional irrigants do not fulfill all of these requisites, besides structurally weakening dentin in the long term. ${ }^{7}$ The other disadvantages of synthetic irrigants are host tissue toxicity, bacterial resistance, questionable and unwanted interactions, and caustic hazard. Hence, extracts of natural products are being tried as an alternative to conventional synthetic ones. The unique advantages of natural substances are ease of availability, long shelf life, and cost-effectiveness besides being safe and biocompatible. ${ }^{3}$

Turmeric (Curcuma longa), neem (Azadirachta indica), and ginger (Zingiber officinale) are edible and also commonly used in traditional medicine for their antimicrobial, anti-inflammatory, and anticarcinogenic properties. ${ }^{3,7-10}$ These are also widely researched and reported in literature. Swallow root (Decalepis hamiltonii) is an edible root widely consumed in the southern parts of India, Sri Lanka, Malaysia, Singapore, and Indonesia. It contains many essential oils and aldehydes and is hence used in Ayurvedic medicine. However, it has been scarcely studied in modern medicine, especially dentistry. ${ }^{11}$

Hence, the aim of the present study was to evaluate the antibacterial effect of $D$. hamiltonii either separately or in
${ }^{1}$ Department of Conservative Dentistry and Endodontics, Madha Dental College (Affiliated to The Tamil Nadu Dr MGR Medical University), Chennai, Tamil Nadu, India

${ }^{2}$ Madha Dental College (Affiliated to The Tamil Nadu Dr MGR Medical University), Chennai, Tamil Nadu, India

Corresponding Author: Anand V Susila, Department of Conservative Dentistry and Endodontics, Madha Dental College (Affiliated to The Tamil Nadu Dr MGR Medical University), Chennai, Tamil Nadu, India, Phone: +91 7550022397, e-mail: drsusila2000@gmail.com

How to cite this article: Susila AV, Sangeeta S. Decalepis hamiltonii (Swallow Root) as a Potential Antimicrobial Agent against Endodontic Pathogens: An In Vitro Study. J Oper Dent Endod 2021;6(1):1-5.

Source of support: Nil

Conflict of interest: None

combination with the extracts of $C$. longa, $A$. indica, and Z. officinale as compared to that of sodium hypochlorite on both standard laboratory strain and clinical isolates of Enterococcus faecalis.

\section{Materials and Methods Preparation of Extracts}

The coarse powder of the roots of C. longa, Z. officinale, and $D$. hamiltonii and leaves of $A$. indica were immersed in the respective solvents, ethanol (for alcoholic extract) or ethanol-water mixture (for hydroalcoholic extract), in individual stoppered containers and allowed to stand for 3 days at room temperature. Then, the mixture was strained and the damp solid material was pressed to yield the crude liquids. These liquids were clarified, filtered, and evaporated in a rotary vacuum evaporator and concentrated to get semisolid mass.

(c) The Author(s). 2021 Open Access This article is distributed under the terms of the Creative Commons Attribution 4.0 International License (https://creativecommons. org/licenses/by-nc/4.0/), which permits unrestricted use, distribution, and non-commercial reproduction in any medium, provided you give appropriate credit to the original author(s) and the source, provide a link to the Creative Commons license, and indicate if changes were made. The Creative Commons Public Domain Dedication waiver (http://creativecommons.org/publicdomain/zero/1.0/) applies to the data made available in this article, unless otherwise stated. 


\section{Microbiological Testing}

The standard laboratory strain of E. faecalis ATCC 292120 and its clinical isolate (obtained from the Microbiology Department which had collected samples from the endodontic patients with symptomatic or asymptomatic irreversible pulpitis) were used in the microbiological assays. Minimum inhibitory concentration (MIC), minimum bactericidal concentration (MBC), and zone of inhibition (ZOI) were determined for the alcoholic and hydroalcoholic extracts of the selected four natural substances. ZOI was measured both individually for the extracts and in different combinations and compared to that of $2.5 \%$ sodium hypochlorite $(\mathrm{NaOCl})$ which acted as a positive control.

E. faecalis ATCC 292120 and the clinical isolate were inoculated in $100 \mathrm{~mL}$ of Mueller Hinton Broth (MHB; HiMedia, India) separately and incubated at $37^{\circ} \mathrm{C}$ overnight. The turbidity was adjusted to $0.5 \mathrm{McF}$ arland standards as recommended by the clinical and laboratory guidelines and the same was followed for preparing lawn culture for the assay.

\section{Broth Microdilution Assay}

Broth dilution is a technique, in which containers holding identical volumes of broth with an antimicrobial solution in incrementally increasing dilutions are inoculated with a known number of bacteria. Broth microdilution denotes the performance of the broth dilution test in a microtiter plate $(500 \mu \mathrm{L}$ capacity).

From the prepared test solutions, doubling dilutions were done from lower dilution to higher dilution in a series of microwell $(33.2,16.6,8.3,4.15,2.07,1.03,0.51,0.25,0.12$, and 0.06) plates (Table 1). The last well contained the negative control with the absence of test reagents. To all the microwell titer plates, $10 \mu \mathrm{L}$ of E. faecalis suspension was added and incubated at $37^{\circ} \mathrm{C}$ overnight. The MIC was recorded as the lowest concentration in the series of dilutions that did not permit the growth (turbidity) of E. faecalis. To substantiate the MIC value, MBC assay was performed.

\section{Minimum Bactericidal Concentration Assay}

The broth microdilution MIC test was extended and confirmed by a subculture to measure the MBC. After incubation of the broth microdilution plate, $5 \mu \mathrm{L}$ of the culture with herbal extracts and the negative control were inoculated on to Mueller Hinton agar plate and incubated overnight at $37^{\circ} \mathrm{C}$. Bactericidal action is indicated by a reduction by at least $99 \%$ in the number of colonies.

Table 1: Dilution percentage and matching concentration of the extracts in $\mathrm{mg} / \mathrm{mL}$

\begin{tabular}{lcc}
\hline Sl. No. & $\%$ & Conc. \\
\hline 1 & 100 & 33.2 \\
2 & 50 & 16.6 \\
3 & 25 & 8.3 \\
4 & 12.5 & 4.15 \\
5 & 6.25 & 2.07 \\
6 & 3.125 & 1.03 \\
7 & 1.0625 & 0.51 \\
8 & 0.53125 & 0.25 \\
9 & 0.265625 & 0.12 \\
10 & 0.1328125 & 0.06 \\
\hline
\end{tabular}

\section{Agar Well Diffusion Assay}

The Mueller Hinton agar plate surface was inoculated by E. faecalis culture adjusted to $0.5 \mathrm{McF}$ carland standards to obtain a cell density of $1.5 \times 10^{8}$ cells $/ \mathrm{mL}$ in $\mathrm{MHB}$ to obtain a lawn culture. Then, a well with a diameter of $8 \mathrm{~mm}$ was punched aseptically with a sterile cork borer and a paper disk saturated with a volume of $100 \mu \mathrm{L}$ of the herbal extract solution at the desired concentration (MIC) was introduced into the well. Then, agar plates were incubated at $37^{\circ} \mathrm{C}$ for 24 hours. The diameter of $\mathrm{ZOI}$ was measured by a graduated scale.

\section{Statistical Analysis}

Students' $t$-test was used to evaluate the intergroup and intragroup differences in MIC, MBC, and ZOI with a confidence interval of $95 \%$ and $p$ value at $\leq 0.05$

\section{Results}

The method of extract preparation (hydroalcoholic/alcoholic) and the nature of the extract (plant source) had a significant effect on both MIC and MBC. MBC was generally higher than MIC.

When tested on ATCC strain, swallow root and turmeric did not show any difference between hydroalcoholic or alcoholic extracts for MIC and MBC values. But neem showed a lower value for hydroalcoholic than alcoholic extract while ginger showed the reverse.

All extracts showed higher MIC and MBC values on clinical isolates than ATCC strain. Neem, turmeric, and ginger showed lower values for hydroalcoholic extract than alcoholic extract. Swallow root showed the same values for both extracts.

Overall, neem had the lowest MIC and MBC of all the extracts, irrespective of the type of extract, or bacterial strain. The highest $\mathrm{MIC}$ and MBC values were found for alcoholic extracts of turmeric and ginger on clinical isolate and hydroalcoholic extract of ginger on ATCC strain. The hydroalcoholic extracts of swallow root, turmeric, and ginger showed the same values on clinical isolate (Fig. 1).

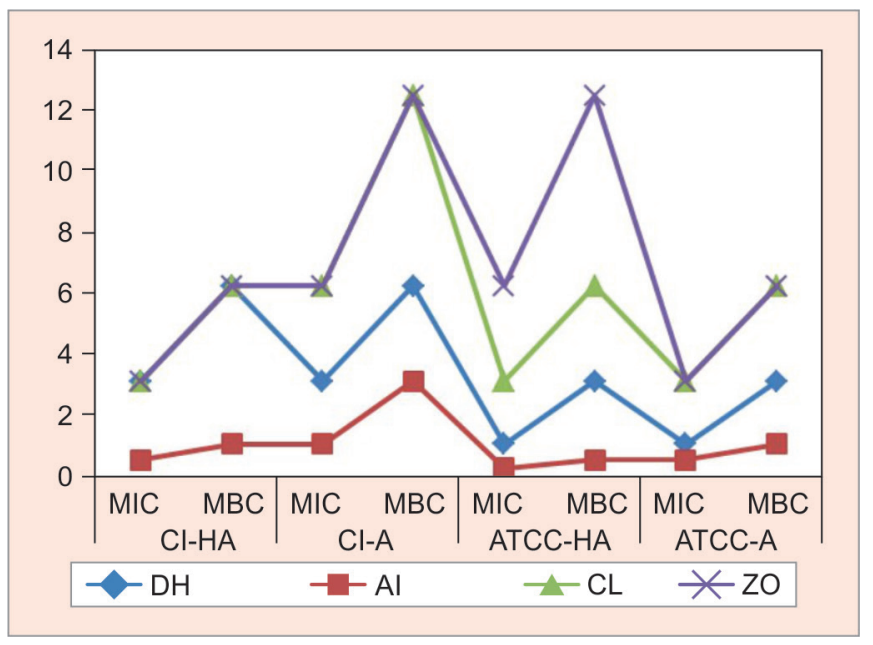

Fig. 1: Antibacterial activity of the test extracts on Enterococcus faecalis. $X$-axis, inhibitory/cidal activity of different types of extracts; $Y$-axis, inhibitory/cidal concentration in $\mu \mathrm{g} / \mathrm{mL}$; $\mathrm{DH}$, Decalepis hamiltonii; $\mathrm{Al}$, Azadirachta indica; $\mathrm{CL}$, Curcuma longa; $\mathrm{ZO}$, Zingiber officinale; $\mathrm{Cl}$, clinical isolate; $\mathrm{HA}$, hydroalcoholic; $\mathrm{A}$, alcoholic 
When testing the extracts individually, in ATCC strain of E. faecalis, hydroalcoholic extract of turmeric showed the smallest ZOI; alcoholic extract of ginger was the largest; hydroalcoholic extract of neem was better than $\mathrm{NaOCl}$ while that of ginger was equivalent; others were inferior to the positive control $\mathrm{NaOCl}$. In clinical isolate of $E$. faecalis, alcoholic extract of ginger was the largest followed by a hydroalcoholic extract of neem; swallow root was equivalent to the positive control $\mathrm{NaOCl}$; others were inferior to the positive control $\mathrm{NaOCl}$ (Table 2). When the combination of extracts was tested, alcoholic extracts of neem + turmeric and neem + swallow root were the smallest; both types of extracts of neem + ginger was either equivalent or superior to the positive control NaOCl; hydroalcoholic extracts of neem + turmeric and neem + swallow root were equivalent to the positive control $\mathrm{NaOCl}$; others were inferior to the positive control $\mathrm{NaOCl}$ (Table 3); however, ZOI for the cocktail of all four test extracts, both hydroalcoholic and alcoholic proved to be larger than the other combinations and the positive control $\mathrm{NaOCl}$ (Table 3).

\section{Discussion}

Endodontic infections are controlled by chemomechanical preparation, intracanal medicament placement, laser disinfection, photodynamic therapy, and ultrasonic agitation of irrigants. The long-term effect of certain conventional irrigants on dentin is far from acceptable and results in structural weakness. Moreover, the safety of some of these irrigants with caustic potential on host periradicular tissues is a cause for concern. Many naturally derived substances are being tried in vitro and in vivo for endodontic disinfection and debridement to overcome these disadvantages of conventional ones.

C. longa, a member of the ginger family contains curcuminoids, that includes mainly curcumin (diferuloyl methane), a yellowcolored pigment; demethoxycurcumin; bisdemethoxycurcumin; and various volatile oils, including turmerone, atlantone, and zingiberone. $^{1-3}$ It is used as a spice, food color, and preservative since time immemorial. It is also used in Ayurveda since 1900 BC for skin, lung, liver, and gastrointestinal (Gl) disorders. Curcumin was first isolated in 1815 by Vogel and Pelletier and its chemical structure was determined in 1910 by J. Milobedzka and V. Lampe. It possesses anti-inflammatory, antioxidant, hepatoprotective, antimicrobial, and anticancer activity. ${ }^{3}$ Curcumin possesses antibacterial properties against a number of gram-positive and gram-negative bacteria. The molecule is pleiotropic and interacts with many molecules during inflammation. It regulates cytokines, transcription factors, protein kinases, adhesion molecules, redox agents, and enzymes. It inhibits bacterial cell division by interacting with FtsZ. A wide range of different concentrations have been found to be safe by Yeon et al. ${ }^{12}$ Bulit et al. showed that it is not toxic to odontoblastlike cells, undifferentiated pulp cells, and human embryonic stem cells. ${ }^{13}$ Mandroli et al. reported a MIC of $333.3 \mu \mathrm{g} / \mathrm{mL}$ against Streptococcus mutans, 125 against Lacticaseibacillus casei, 167 against Actinomyces viscosus, 208 against Prevotella intermedia, 125 against Porphyromonas gingivalis, and no activity against $E$. faecalis. ${ }^{2}$ However, the results of another study by Neelakantan et al. were contradictory in that a significant antibacterial activity was seen compared to $\mathrm{NaOCl}^{14}$ Another study by Vinothkumar et al., using quantitative polymerase chain reaction (QPCR), concurred with the superior efficacy of turmeric compared to Aloe vera. ${ }^{15}$

Curcumin was shown to prevent the biofilm formation by eliminating extracellular polymeric substances by Kishen et al. ${ }^{16}$

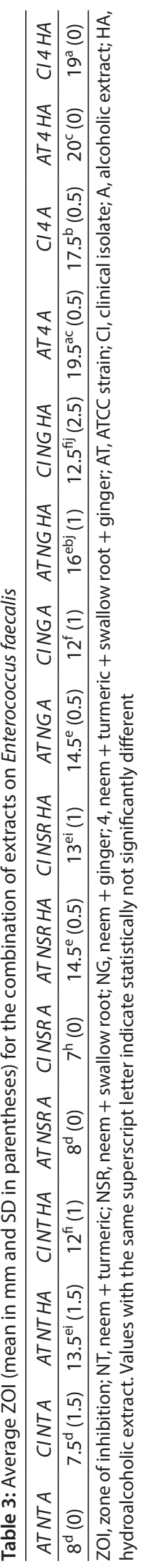


Hemanshi Kumar showed that curcumin produced greater ZOI against anaerobes like E. faecalis. ${ }^{9}$ Another study showed equivalent activity of turmeric and $\mathrm{NaOCl}$ superior to chlorhexidine (CHX) on $E$. faecalis biofilm in extracted teeth. ${ }^{17}$ It was able to eliminate E. faecalis biofilm when used as an intracanal medicament (ICM), better than calcium hydroxide $\left(\mathrm{Ca}(\mathrm{OH})_{2}\right)$ but less than $2 \% \mathrm{CHX}$ in a study by Yadav et al. ${ }^{18}$ However, Pandey et al. showed that it is inferior to $5.25 \% \mathrm{NaOCl}$ on E. faecalis biofilms. ${ }^{19}$ In the present study also, turmeric had higher MIC and MBC when the alcoholic extract was used on clinical isolate. Its ZOI was also the smallest.

A. indica, known as the Indian neem/margosa tree, belongs to Meliaceae family. The chemical components present are nimbin, nimbinene, 6-desacetylnimbinene, nimbandiol, ascorbic acid, n-hexacosanol and amino acid, 7-desaacetyl-7-benzoylazadiradione, 7-desacetyl-7-benzoylgedunin, 17-hydroxyazadiradione, and nimbiol $^{20}$ which act as biocompatible antioxidant, antiviral, antifungal, antibacterial, and anticarcinogenic agents. ${ }^{3}$ Subapriya et al. also reported similar properties. ${ }^{21}$ In a study by Maina et al., it was found that the MIC of aqueous extract was $50 \mu \mathrm{g} / \mathrm{mL}$ and it inhibited $46 \%$ of $E$. faecalis at $24 \mathrm{~h}$ and $85 \%$ at $48 \mathrm{~h}$. However, the ethanolic extract had a MIC of $0.1 \mu \mathrm{g} / \mathrm{mL}$ and inhibited $88 \%$ at $24 \mathrm{~h}$ and $96 \%$ at $48 \mathrm{~h}$. The MBC was not detectable for aqueous extract while it was $6.25 \%$ for ethanolic extract. ${ }^{7}$ This concurs with the findings of the current study.

Arati et al. found that the ethanolic extract of neem has significant antimicrobial activity against $E$. faecalis. ${ }^{22}$ Hannah et al. ${ }^{23}$ found that it is better than $\mathrm{NaOCl}$ in preventing bacterial adherence. Along with curcumin, it has better antimicrobial activity than other herbal extracts in a study by Vinothkumar et al. ${ }^{15}$ However, in the present study, this combination was less effective than others. Bohora et al. found that both aqueous and alcoholic extracts were as effective as $\mathrm{NaOCl}^{8}$ on a mixed culture of $E$. faecalis and Candida albicans. At a conc. of $25 \%$, it was able to remove biofilm of $E$. faecalis to a limited extent only unlike $3 \% \mathrm{NaOCl}$ which completely eliminated $E$. faecalis in a study.$^{24}$ In the present study, $\mathrm{MIC}$ and MBC were the lowest for neem when its hydroalcoholic extract was used, especially on ATCC. It also showed the largest ZOI for the hydroalcoholic extract on both ATCC and clinical isolate. Combining other natural extracts like ginger and swallow root with neem showed an additive effect.

Z. officinale, ginger, is used in traditional medicine for treating many diseases. Ginger's pungent components offer powerful anti-inflammatory and antioxidant activities, making it useful in arthritis, Alzheimer's disease, cancer, and cardiovascular disease. It contains zingibain, an enzyme that counteracts inflammation. The active compounds of ginger are divided into two groups: volatile essential oils and fragrant or harsh phenol compounds. ${ }^{25}$ Among these, volatile essential components, which constitute zingerone and shagelol, were reported to be responsible for their antibacterial properties. ${ }^{10}$ Its ethanolic extract was found to be effective against $C$. albicans and E. faecalis by Atai et al. ${ }^{26}$ Similar results were reported by Gulve et al. ${ }^{27}$ Gingerol in ginger was found to act on P. gingivalis, Porphyromonas endodontalis, and $P$. intermedia by Park et al. ${ }^{28}$ It was found to act on bacterial endotoxin in a study by Maekawa et al. ${ }^{29}$ In the present study, MIC of ginger was the highest when the hydroalcoholic extract was used against ATCC or when the alcoholic extract was used against clinical isolate of E. faecalis. However, its ZOI was the largest for its alcoholic extract when used on both ATCC and clinical isolate.
Roots of $D$. hamiltonii are edible and used for their healthpromoting properties in traditional cuisine. They contain aldehydes, amyrins, lupeols, and volatile flavor compounds such as 2-hydroxy-4methoxybenzaldehyde; vanillin; and essential oils like methylresorcylaldehyde, atlantone, terpinene, and geraniol. A combinational molecule containing peptic polysaccharide with bound phenolics identified in the roots of $D$. hamiltonii and its breakdown products have been known to have health beneficial properties. It was found to have a maximum $\mathrm{ZOI}$ of $24 \mathrm{~mm}$ against E. faecalis intermediate between those of ginger and $0.2 \% \mathrm{CHX}$. It showed a MIC of $2.5 \mathrm{mg} / \mathrm{mL} .{ }^{11}$ In the present study, swallow root had higher MIC and MBC when used on clinical isolate of $E$. faecalis which was similar to that of turmeric. Its ZOI was moderate and comparable to $\mathrm{NaOCl}$. However, it should be noted that in the present study, ZOI was determined for MIC of the extracts, while many studies cited above have used maximum inhibitory concentration.

E. faecalis, a facultative anaerobe, is frequently used in biofilm models to test the potential antimicrobial actions of new medicaments, irrigants, irrigating protocols, instrumentation techniques, irrigant activation, sealers, and obturating materials in endodontics. The reason behind such practice is because it is one of the important organisms in endodontic infection and reinfection. It is commonly detected in asymptomatic, persistent endodontic infections and possesses virulence factors like lytic enzymes, cytolysin, aggregation substance, pheromones, and lipoteichoic acid. It can survive by genetic polymorphism and it has the ability to bind to dentin, invade dentinal tubules, and survive starvation. It has been shown that it adheres to host cells, express proteins that allow it to compete with other bacterial cells, and alter host responses. Moreover, it suppresses the action of lymphocytes, potentially contributing to endodontic failure. ${ }^{4}$ Several studies showed that E. faecalis was found more in the cases of failed endodontic treatment than in the cases with primary infections. ${ }^{30}$ Thus, in the present study both standard laboratory strain and clinical isolate of $E$. faecalis were used as the microorganism to test the effect of plant extracts.

In this study, $2.5 \% \mathrm{NaOCl}$ was used as the positive control as this concentration of the irrigant is widely used in endodontic practice than the other concentrations, and hence, the results simulate a routine clinical scenario. Further, this irrigant is considered as a gold standard for testing any new or potential ones, as it possesses a time-tested effect on a wide spectrum of microbes and tissue dissolution efficacy.

Phytochemicals and nutrients exert additive and synergistic effects when used in the right combination. Similarly, a wrong combination can exert an inhibitive or negative effect. Thus, in this study, the effects of combining four different plant extracts on endodontic pathogen were investigated and it appears that neem and turmeric may have a negating effect if used together, when other plant extracts are added, they can produce a significant positive effect. Exploring such combinations may be useful in choosing the best combination for the desired effect in clinical microbiology.

One limitation of this study is its design being in vitro and the fact that the biocompatibility of this extract of $D$. hamiltonii is not yet reported. However, since it is an edible root used in Asian cuisine for centuries, it may be probable that it is also safe like the ginger and turmeric family which has many similarities. Future studies can focus on this aspect and test the efficacy in clinical studies before it can be recommended as an alternative to conventional irrigants. 


\section{CONCLUSION}

The potential for using the extract of swallow root for antimicrobial action was tested in this study. This edible root, similar to ginger, was found to be having satisfactory antimicrobial action against both standard strain and clinical isolate of E. faecalis. Hence, it has the potential to be used as an alternative to conventional irrigants and medicaments in endodontics. The combination cocktail of the four natural extracts proved to be more effective than the positive control of $2.5 \% \mathrm{NaOCl}$.

\section{HighLights}

- Extracts of natural substances have the potential for being safer and effective alternatives to synthetic ones in endodontic disinfection and cleaning

- D. hamiltonii (swallow root) has similar potential as C. longa in inhibiting E. faecalis

- Swallow root extract can be tried as an alternative to sodium hypochlorite in endodontic irrigation.

\section{ACKnowledgment}

Authors would like to thank Dr Mahalakshmi K, MSc, PhD, Department of Microbiology, Sree Balaji Dental College, Bharath University, Chennai, Tamil Nadu, India, 600100, for her help in the microbiological assays.

\section{References}

1. Aggarwal B, Sung B. Pharmacological basis for the role of curcumin in chronic diseases: an age-old spice with modern targets. Trends Pharmacol Sci 2008;30(2):85-94. DOI: 10.1016/j.tips.2008.11.002.

2. Mandroli P, Bhat K. An in-vitro evaluation of antibacterial activity of curcumin against common endodontic bacteria. J Appl Pharm Sci 2013;3(10):106-108. DOI: 10.7324/JAPS.2013.31018.

3. Jain $P$, Ranjan $M$. Role of herbs in root canal irrigation-a review. IOSR J Pharm Biol Sci 2014;9(2):6-10. DOI: 10.9790/3008-09260610.

4. Sinha $D$, Sinha A. Natural medicaments in dentistry. AYU 2014;35(2):113-118. DOI: 10.4103/0974-8520.146198.

5. Byström A, Sundqvist G. Bacteriologic evaluation of the efficacy of mechanical root canal instrumentation in endodontic therapy. Scand J Dent Res 1981;89(4):321-328. DOI: 10.1111/j.1600-0722.1981. tb01689.x.

6. Siqueira Jr J, Rocas I, Favieri A, et al. Chemomechanical reduction of the bacterial population in the root canal after instrumentation and irrigation with $1 \%, 2.5 \%$, and $5.25 \%$ sodium hypochlorite. J Endod 2000;26(6):331-338. DOI: 10.1097/00004770-20000600000006.

7. Maina S, Dimba E, Oyugi J, et al. Antimicrobial efficacy of Azadirachta indica (Neem) twigs aqueous and ethanol extracts on tooth root canals biofilms. Int J Pharmacognosy Phytochem Res 2015;7(4): 735-739.

8. Bohora A, Hegde V, Kokate S. Comparison of the antibacterial efficiency of neem leaf extract and $2 \%$ sodium hypochlorite against $E$. faecalis, C. albicans and mixed culture-an in vitro study. Endodontology 2010;22:8-12.

9. Kumar H. An in vitro evaluation of the antimicrobial efficacy of Curcuma longa, Tachyspermum ammi, chlorhexidine gluconate, and calcium hydroxide on Enterococcus faecalis. J Conserv Dent 2013;16(2):144-147. DOI: 10.4103/0972-0707.108197.

10. Giriraju A, Yunus G. Assessment of antimicrobial potential of $10 \%$ ginger extract against Streptococcus mutans, Candida albicans, and Enterococcus faecalis: an in vitro study. Indian J Dent Res 2013;24(4):397-400. DOI: 10.4103/0970-9290.118356.
11. Arunkumar M, Tejaswi B, Susila V. Anti bacterial and anti inflammatory efficacy of Zingiber officinale and Decalepis hamiltonii - in vitro study. Int J Drug Develop Res 2014;6(3):133-137.

12. Yeon K, Kim S, Kim Y. Curcumin produces an antihyperalgesic effect via antagonism of TRPV1. J Dent Res 2010;89(2):170-174. DOI: 10.1177/0022034509356169.

13. Bulit F, Grad I, Manoil D. Antimicrobial activity and cytotoxicity of 3 photosensitizers activated with blue light. J Endod 2014;40(3): 427-431. DOI: 10.1016/j.joen.2013.12.001.

14. Neelakantan P, Subbarao C, Subbarao C. Analysis of antibacterial activity of curcumin against Enterococcus faecalis. Int J Curr Res Rev 2011;3(9):37-42.

15. Vinothkumar T, Rubin M, Balaji L, et al. In vitro evaluation of five different herbal extracts as an antimicrobial endodontic irrigant using real time quantitative polymerase chain reaction. J Conserv Dent 2013;16(2):167-170. DOI: 10.4103/0972-0707.108208.

16. Kishen A, George S, Kumar R. Enterococcus faecalis mediated biomineralized biofilm formation on root canal dentine in vitro. J Biomed Mater Res A 2006;77(2):406-415. DOI: 10.1002/jbm.a.30622.

17. Neelakantan P, Subbarao C, Sharma S, et al. Effectiveness of curcumin against Enterococcus faecalis biofilm. Acta Odontol Scand 2013;71(6):1453-1457. DOI: 10.3109/00016357.2013.769627.

18. Yadav R, Tikku A, Chandra A, et al. A comparative evaluation of the antimicrobial efficacy of calcium hydroxide, chlorhexidine gel, and a curcumin based formulation against Enterococcus faecalis. Natl J Maxillofac Surg 2018;9(1):52-55. DOI: 10.4103/njms.NJMS_47_17.

19. Pandey S, Shekhar R, Paul R, et al. A comparative evaluation and effectiveness of different antimicrobial herbal extracts as endodontic irrigants against Enterococcus faecalis and Candida albicans-an in vitro study. Univ J Dent Sci 2018;4:75-81.

20. Singh $\mathrm{H}$, Kaur M, Dhillon J, et al. Neem: a magical herb in endodontics. Stomatological Dis Sci 2017;1(2):50-54. DOI: 10.20517/25730002.2016.10.

21. Subapriya R, Nagini S. Medicinal properties of neem leaves: a review. Curr Med Chem Anticancer Agents 2005;5(2):149-156. DOI: 10.2174/1568011053174828.

22. Arathi N, Ranganath N, Soumya G, et al. Evaluation of antibacterial and anticandidial efficacy of aqueous and alcoholic extract of neem (Azadhiracta indica)-an in vitro study. Int J Res Ayurveda Pharm 2011;2(1):230-235.

23. Rosaline H, Kandaswamy D, Gogulnath D, et al. Influence of various herbal irrigants as a final rinse on the adherence of Enterococcus faecalis by fluorescence confocal laser scanning microscope. J Conserv Dent 2013;16(4):352-355. DOI: 10.4103/0972-0707.114365.

24. Panchal V, Gurunathan D, Muralidharan N. Comparison of antibacterial efficacy of cinnamon extract, neem extract as irrigant and sodium hypochlorite against Enterococcus faecalis: an in vitro study. Indian J Dent Res 2020;31(1):124-128. DOI: 10.4103/ijdr.IJDR_177_18.

25. Rahman S, Thangaraj S, Salique S, et al. Antimicrobial and biochemical analysis of some spices extract against food spoilage pathogens. Internet J Food Saf 2010;12:71-75.

26. Atai Z, Atapour M, Mohseni M. Inhibitory effect of ginger extract on Candida albicans. Am J Appl Sci 2009;6(6):1067-1069. DOI: 10.3844/ ajassp.2009.1067.1069.

27. Gulve M, Gulve N. Comparison of antimicrobial efficacy of ginger extract and 2\% sodium hypochlorite against Enterococcus faecalis using agar diffusion method. J Indian Dent Assoc 2010;4:347-349.

28. Park M, Bae J, Lee D. Antibacterial activity of [10]-gingerol and [12]-gingerol isolated from ginger rhizome against periodontal bacteria. Phytother Res 2008;22(11):1446-1449. DOI: 10.1002/ ptr.2473.

29. Maekawa L, Valera M, de Oliveira L, et al. Effect of Zingiber officinale and propolis on microorganisms and endotoxins in root canals. J Appl Oral Sci 2013;21(1):25-31. DOI: 10.1590/1678-7757201302129.

30. Alghamdi F, Shakir M. The influence of Enterococcus faecalis as a dental root canal pathogen on endodontic treatment: a systematic review. Cureus 2020;12(3):e7257. DOI: 10.7759/cureus.7257. 